\title{
Wearable Activity Trackers and Older Adults: The Social Effect and Importance in Healthcare
}

Jessica DeClercq ${ }^{*}$; Shelia R Cotten ${ }^{*}$; Wei Peng ${ }^{*}$; Anastasia Kononova ${ }^{*}$; Marie Bowen ; Kendra Kamp*; R.V Rikard*; Lin $\mathrm{Li}^{*}$

Michigan State University, East Lansing, MI, United States

*all authors contributed equally

\section{Corresponding Author:}

Marie Bowen

Michigan State University

220 Trowbridge Rd

East Lansing, MI, 48824

United States

Phone: 15173551855

Email: stuvemar@msu.edu

\begin{abstract}
Background: Many older adults develop chronic diseases, such as heart disease and diabetes, which are correlated with low levels of physical activity. Chronic diseases can result in a decreased quality of life, increased health care costs, and premature mortality. Adults, specifically older adults, who started using wearable activity trackers (WATs) have exhibited an increase in daily activity levels. Although WAT use has increased, only 7\% of older adults use a WAT. The use of WATs has the potential to facilitate chronic condition self-management, with patients engaging in personalized care and health care providers receiving accurate data about patient physical activity. One benefit of WATs is the opportunity to develop social relationships. Social relationships have as much impact on physical health as physical activity. Older adults with larger networks show higher levels of health.
\end{abstract}

Objective: The purpose of this study is to explore how WATs connect older adults to those around them and to determine the benefits of sharing WAT data with healthcare providers.

Methods: Ten focus groups and 20 interviews were conducted with older adults who had varying levels of WAT use. Each participant was categorized as one of the following; long-term user (used WATs for six months or more); short-term user (used WATs for less than six months); former user; never user. Discussion topics included WAT social aspects, the frequency and benefits of sharing individual WAT data with healthcare providers, and strategies to increase the number of long-term WAT users among older adults.

Results: Preliminary data suggests that WATs have the potential to better connect people socially through their competition and gamification aspects. Trackers are able to connect numerous people together and turn an individual's health journey into an engaging and communal game. Some older adults also reported taking their WAT data to their healthcare provider. Sharing the WAT data made many of them feel like they were taking charge of their own health. The features that were reported as most commonly talked about with providers were sleep patterns, steps taken, and heart rate. A difference between the long-term and former users studied was their level in social interaction. Preliminary data suggests that more long-term users reported sharing their data with others than former users.

Conclusions: Initial analysis suggests that WAT users can benefit more from social interactions with their WATs. Tracking activity with others holds a person accountable and can make it more enjoyable. Sharing WAT data with healthcare providers has been suggested to comfort older adults by making them feel more in control of their life. Older adults can potentially talk with their doctors more intelligently about their activity levels through their WAT.

(iproc 2017;3(1):e50) doi: 10.2196/iproc.8580

\section{KEYWORDS}

chronic illness; health; healthcare; obesity; Older adults; social influence 


\section{Multimedia Appendix 1}

Full poster.

[PDF File (Adobe PDF File), 388KB-Multimedia Appendix 1]

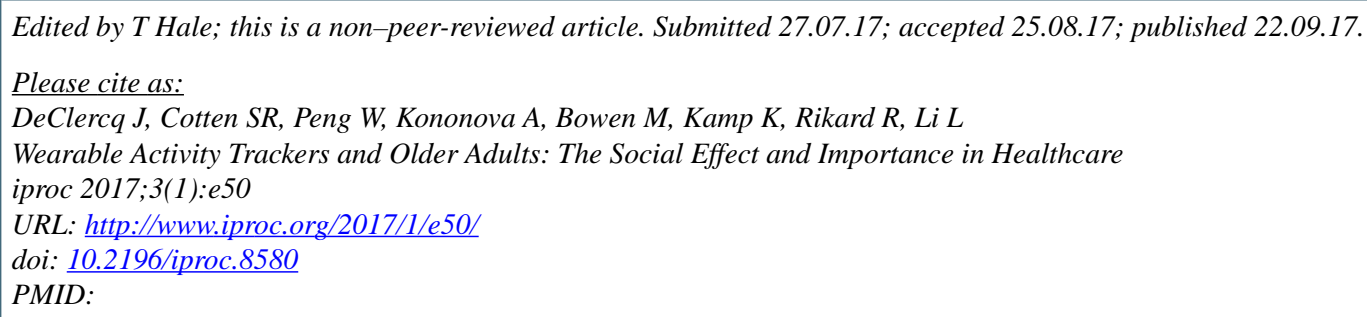

(CJessica DeClercq, Shelia R. Cotten, Wei Peng, Anastasia Kononova, Marie Bowen, Kendra Kamp, R.V. Rikard, Lin Li. Originally published in Iproceedings (http://www.iproc.org), 22.09.2017. This is an open-access article distributed under the terms of the Creative Commons Attribution License (https://creativecommons.org/licenses/by/4.0/), which permits unrestricted use, distribution, and reproduction in any medium, provided the original work, first published in Iproceedings, is properly cited. The complete bibliographic information, a link to the original publication on http://www.iproc.org/, as well as this copyright and license information must be included. 